\title{
Inclusión, atención a la diversidad y neuroeducación en Educación Física Inclusion, attention to diversity and neuroeducation in physical education
}

\author{
*Antonio Hernández Fernández, **Claudia De Barros Camargo \\ *U niversidad de Jaén (España), **U niversidad de Granada(España)
}

\begin{abstract}
Resumen. El presente trabajo tiene como objetivo general analizar la relación entre inclusión, atención a la diversidad y neuroeducación en el ámbito de Educación Física, a través del análisis factorial confirmatorio (AFC) de una escala, creada ad hoc, validada y confirmada. Para la búsqueda de respuestas, se ha realizado un proceso de investigación no experimental, descriptivo y explicativo. El instrumento de recogidade datos hasido una escala Likert (con número impar de opciones, cinco niveles de respuestay rango de 1 a5) que se ha val idado en contenido y fiabilidad (al pha de Cronbach, .833) buena. Lavalidez de constructo se ha realizado con un análisis factorial exploratorio (AFE) (KM 0: .771, Bartlett: sign.000, determinante: $\left.6.064 \mathrm{E}^{-7}\right)$. La muestra ha sido de 359 estudiantes universitarios que cursan la especialidad de Educación Física. El AFC nos permitió concluir que es posible esta relación, con mayor fuerza entre inclusión y atención a la diversidad, y entre estaúltima y neuroeducación, y con menor fuerza entre inclusión y neuroeducación. (X²/ gl (3.09). IAA: GFI (.85), RMSEA (.076), NCP (437.63), SRMR (.44), RMS (.061), ECVI (2.31). IAI: AGFI (.79), IFI (.96), NFI (.94), TLI O NNFI (.94), RFI (.92), CFI (.96). IP: PNFI (.71), PGFI (.60), AGFI (.79)).
\end{abstract}

Palabras Clave: educación inclusiva, diversidad, neurociencia, neuroeducación, educación física.

\begin{abstract}
The general objective of this work is to analyze the relationship between inclusion, attention to diversity and neuroeducation in the field of Physical Education, through the confirmatory factor analysis (CFA) of a scale, created ad hoc, validated and confirmed. For the search for answers, a non-experimental, descriptive and explanatory research process has been car ried out. The data collection instrument was a Likert scale (with an odd number of options, five response levels and a range from 1 to 5) that was validated for content and reliability (Cronbach's alpha, .833). The construct validity has been carried out with an exploratory factor analysis (EFA) (KM 0 : .771, Bartlett: sign.000, determinant: 6.064E-7). The sample consisted of 359 university students majoring in Physical Education. The CFA allowed us to conclude that this relationship is possible, with greater strength between inclusion and attention to diversity, and between the latter and neuroeducation, and with lesser strength between inclusion and neuroeducation. (X2/ gl (3.09). IAA: GFI (.85), RMSEA (.076), NCP (437.63), SRMR (.44), RMS (.061), ECVI (2.31). IAI:AGFI (.79), IFI (.96), NFI (.94), TLI OR NNFI (.94), RFI (.92), CFI (.96). IP: PNFI (.71), PGFI (.60), AGFI (.79)).
\end{abstract}

Keywords: inclusive education, diversity, neuroscience, neuroeducation, physical education.

\section{Introducción}

La inclusión educativa, está adquiriendo importancia en nuestra sociedad y se está convirtiendo en un tema de interés y necesidad a nivel social y educativo (Muntaner, 2019). Ya que todo el alumnado, sin importar sus habilidades, capacidades y aptitudes, tiene derecho a recibir una educación inclusiva de calidad y participar en ella (Echeita \& Ainscow, 2011; Arnaiz, 2011; Ainscow, 2017). En opinión de Booth \& Ainscow (1998) y más actualmente con Arnaiz (2020) y Palomares (2020), lanoción de inclusión comprendedos conceptos básicos: el de la comunidad y el de participación. Ambos se caracterizan por su conexión con los procesos de

\footnotetext{
Fecharecepción: 15-11-20. Fecha de aceptación: 24-01-21 Antonio Hernández Fernández ahernand@ ujaen.es
}

inclusión, y el carácter de proceso atribuido a la misma. Por tanto, la educación inclusiva se propone aumentar la participación de todos los estudiantes en el currículum de la escuela, y la reducción de la exclusión escolar y social. Numerosos estudios han mostrado que el área deEducación Físicaes una herramienta fundamental que favorece la inclusión del alumnado con necesidades específicas de apoyo educativo (Kinio, Dufresne, Brandys $\&$ Jetty, 2017; Flores, 2019).

La educación inclusiva representa el elemento base de esta investigación, la relación con la atención a la diversidad y la neuroeducación es el objetivo que nos planteamos, con la certeza de que esta interrelación, aportarán nuevas perspectivas en la inclusión educativa. El alumnado con necesidades específicas de apoyo educativo asiste a centros ordinarios, por ello es necesario pensar y crear propuestas educativas inclusivas para fa vorecer su desarrollo en el ámbito educativo. Así el 
área de Educación Física se caracteriza por favorecer la inclusión con una metodología adecuada (Segura \& Pa rra, 2019). Cada vez hay más interés sobre la atención a la diversidad en las clases de Educación Física, por ello, es necesario que el profesorado de esta área muestre una actitud positiva y estéformado en inclusión (A bellán, 2015; Hernández, Labrador, Niort, Berbel \& Trullols, 2017). De esta forma se plantea una Educación Física que posibilite el desarrollo de la persona en comunidad. Para que, de esta manera en los espacios educativos, el alumnado sea propositivo, participativo, autónomo, consciente y reflexivo del hacer, pensar, y actuar (Pastas, 2018).

Dentro de los métodos pedagógicos más recientes en la actualidad aparece inmersa una nueva ciencia, la N euroeducación entendida como una novedosa línea de pensamiento y acción cuyo objetivo primordial es acercar a los educadores los conocimientos relacionados con el cerebro y el aprendizaje, en un intento de unión entre Pedagogía, Psicología Cognitiva y N eurociencias (Campos, 2010). Neuroeducación es, pues, una nueva interdisciplina y transdisciplina que promueve una ma yor integración de las ciencias de la educación con aquellas que se ocupan del desarrollo neurocognitivo de la persona humana (Battro \& Cardinali, 1996). Interdisciplina en tanto es la intersección de muchas disciplinas relacionadas con el aprendizaje y la enseñanza en todas sus formas, transdisciplina en cuanto es una nueva integración, absolutamente original de aquellas en una nueva categoría conceptual y práctica. Koizumi (2006) y Maureira (2018) nos dicen que uno delos desafíos del siglo XXI es que los educadores deban conocer el fenómeno de aprendizaje y que esto es solo posible a través de los procesos neurales, estructuras del cerebro y cómo las neuronas y las sinapsis están relacionadas con el aprendizaje. También indica que es necesario incluir la asignatura de neuroeducación (entendiendo como el puente entre la neurociencia y educación) en todas las carreras de pedagogía de manera que los futuros docentes posean herramientas para generar ambientes óptimos de aprendizaje, de igual forma en el sector de la investigación en los últimos años se han desarrollado numerosos trabajos sobre aspectos de la neuroeducación aplicados a la educación física, así destacamos los estudiossobre inteligenciaemocional (M ujica, 2021; M ujica \& O rellana, 2020; M elguizo, Zurita, U bago \& González, 2020), con lo que no tardarán en surgir más experiencias sobre redes neuronales, neuronas espejo, etc.

Según Flores y Maureira (2020) un argumento interesante para incluir la neurociencia en la formación de los futuros profesionales de la educación es que actualmente existen diversos «neuromitos», debido a que el profesorado (sin formación en neurociencia en su ca rrera pedagógica) ante la inquietud del conocimiento leen documentos que no poseen ninguna clase de revisión científica y por ende aprenden e informan erróneamente a sus pares. Estamos de acuerdo con los autores antes mencionados, en que se necesita argumentos en la disciplina de Educación Física, ya que, desde otras áreas sólo la ven como la instancia recreativa de los educandos y no consideran todos los beneficios en los procesos cognitivos y de aprendizaje para las otras ma terias 0 asignaturas (Tenorio, 2013), es por ello que los futuros profesionales deben conocer para investigar, deben tener claro que el ejercicio no sólo conlleva beneficios físicos y de rendimiento, también colabora con la mejora de las funciones cognitivas como la atención, memoria a corto plazo, etc.

Dentro delaeducación inclusivay laneuroeducación tenemos un aspecto importante a tratar, como es la diversidad. "Se entiende por diversidad todas aquellas características excepcionales del alumnado, provocadas por diversos factores que requieren una atención especializada para que todos los estudiantes al cancen un mismo nivel de aprendizaje» (Silva, 2007, p. 3). Esta diversidad se traduce en los centros educativos en dificulta des de aprendizaje, discapacidades físicas, psíquicas y sensoriales, los grupos de riesgo, las minorías étnicas, etc.

En los últimos años tenemos investigaciones y experiencias de relevancia sobre la atención a la diversidad y la toma de medidas en el ámbito de la educación física, algunos ejemplos los encontramos en González-López \& M acías-García (2018) sobre discapacidad física, o bien 0 choa-M artínez, Hall-López, Carmona López, Reyes Castro, Sáenz-López, \& Conde García (2019) en discapacidad auditiva o sobre identidad de género en Pérez-Enseñat, \& M oya-Mata (2020). Estas y otras discapacidades requieren unas medidas de atención por parte de docentes y autoridades educativas, las cuales no siempre tienen carácter inclusivo (centros específicos de educación especial) o pueden carecer de aspectos neuroeducativos en su concepción, ya tenemos investigaciones sobre la formación docente y atención a la diversidad que muestran carencias en la misma (Valencia-Peris, M ínguez-Alfaro, \& M artos-García, 2019).

De este modo, como se puede apreciar a lo largo de esta introducción, no se encuentran en la literatura estudios que de manera conjuntan interrelacionen inclusión educativa, neuroeducación y medidas de atención a 
la diversidad, surgiendo la investigación que aquí se presenta.

\section{M etodología}

Esta investigación tiene el siguiente objetivo general: analizar la relación entre inclusión, atención a la diversidad y neuroeducación en el ámbito de Educación Física. Partimos de un diseño no experimental, descriptivo y explicativo. La metodología seleccionada es de tipo cuantitativo, tomando como referencia un paradigma interpretativo (Guba, 1990; Ricoy, 2006). Para la realización de la investigación se opta por la utilización de una escala Likert de 30 ítems, con número impar de opciones, cinco niveles de respuesta y rango de 1 a 5, como instrumento de investigación.

\section{Participantes}

La población está constituida por los estudiantes de último curso del Grado de Educación Primariay Educa ción Social que cursan o han cursado la materia de Educación Física (418 sujetos) y que tomaremos en su tota lidad. La escala Likert fue respondida finalmente por 359 participantes.

\section{Dimensiones y variables}

Las dimensiones que consideramos en este estudio, extraídas del marco teórico y la construcción de la esca la Likert son: A.-Inclusión educativa, B.-Medidas de atención a la diversidad, C.-N euroeducación. En consecuencia, establecemos como variable independiente: Educación Física. Las variables dependientes son: inclusión educativa, medidas de atención a la diversidad y neuroeducación.

\section{Instrumento}

Utilizamos una escala Likert con 30 ítems, construida a través de una tabla de operacionalización.

El diseño de la escala Likert se ha realizado con una tabla de operacionalización (tabla 1), por otra parte, la validación se ha realizado, en un primer momento de contenido, con un juicio de expertos y prueba piloto, en segundo lugar, se realizó un análisis factorial para validar la escala en su constructo utilizándose como software SPSS v25. El anál isis de fiabilidad se realizó cal culando el al pha de Cronbach, dando una puntuación de .833, que se considerabueno (George\& Mallery, 2003), para el modelaje estructural se ha utilizado Lisrel 8.80.

\section{Análisis de datos}

Validez de contenido

Respecto de la validez, en primer lugar, se procedió a una validez de contenido que se realizó por quince especial istas doctores autorizados para real izar esta evaluación y pertenecientes a diferentes universidades. Se

Tabla 1.

Tabla de operacionalización

\begin{tabular}{|c|c|c|}
\hline Dimensiones & Variables & Ittems. \\
\hline $\begin{array}{l}\text { A. - Inclusión } \\
\text { educativa. }\end{array}$ & $\begin{array}{l}\text { Inclusión } \\
\text { educativa. }\end{array}$ & $\begin{array}{l}\text { A1.-La inclusión educativa es el proceso de análisis sistemático de las culturas, las politicas y las prácticas escolares para tratar eliminar o minimizar, a través de iniciativas } \\
\text { sostenidas de mejora e innovación escolar. } \\
\text { A2.-LaActividad FísicaAdaptada es la que se acondiciona para per sonas con condiciones limitadas como la discapacidad, las deficiencias en la salud o la edad avanzada, para } \\
\text { favorecer la integración de estas per sonas en un estilo de vida saludable a través de la práctica deportiva. } \\
\text { A6.-La práctica del D eporte y de laActividad Física en grupo inclusivo es aquella en la cual las per sonas con discapacidad compartirán todas las actividades físico- } \\
\text { deportivas con las personas sin discapacidad. } \\
\text { A7.-La práctica de Deporte y deActividad Física en grupos específicos/ exclusivos, hace referencia a la práctica de las per sonas con discapacidad, real izando un trabajo } \\
\text { conjunto, al margen del grupo ordinario. } \\
\text { A9.-La actividad física adaptaday los deportes adaptados son actual mente un contenido obligatorio en todos los diseños de grado. }\end{array}$ \\
\hline & $\begin{array}{l}\text { aM edidas de } \\
\text { atención a la } \\
\text { diversidad. }\end{array}$ & 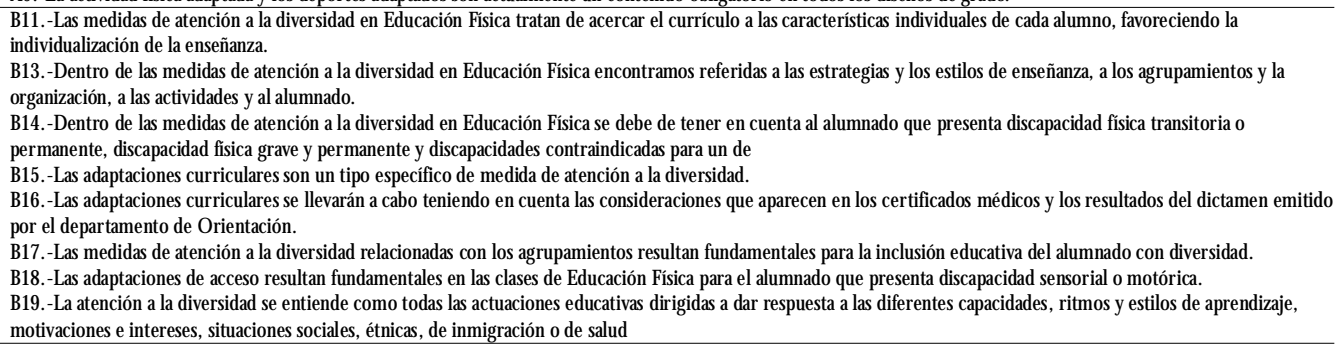 \\
\hline n. & $\mathrm{Oe}$ & $\begin{array}{l}\text { C20.-La neuroeducación es una línea de pensamiento que tiene por objeto unir los conocimientos del cerebro y el aprendizaje con la psicología cognitiva y la pedagogía. } \\
\text { C21.-La disciplina neuroeducación física per mite establecer relaciones entre los conocimientos de la neurociencia y la actividad motriz. } \\
\text { C22.-Entre los diferentes aspectos que estudia la neuroeducación física encontramos: la relación entre actividad física y la disminución de la ansiedad y la depresión, la } \\
\text { relación entre ejercicio físico y autoestima, o la mejora la de los procesos atención. } \\
\text { C23.-M ovimiento, pensamiento y conducta están íntimamente relacionados. } \\
\text { C24.-Existe relación entre actividad físicay desar rollo de habilidades cognitivas. } \\
\text { C25.-Las clases de Educación Física se convierten en un entorno natural adecuado para observar problemas neuronales asociados al área motriz. } \\
\text { C26.-Tener conocimientos sobre el control neuronal del movimiento ayudará a los maestros/ as especialistas de Educación Física a programas sesiones para al umnado que } \\
\text { presenta diversidad funcional. } \\
\text { C27.- Tener conocimientos sobre el control neuronal del movimiento ayudaráa los maestros/ as especial istas de Educación Física a programas sesiones para alumnado que } \\
\text { presenta parálisis cerebral. } \\
\text { C28.-La programación de sesiones en Educación Física con base en los conocimientos aportados por la neuroeducación tiene una repercusión positiva en la inclusión del } \\
\text { alumnado. } \\
\text { C29.-La formación en neuroeducación física per mite a los docentes comprender mejor la situación de las per sonas con diversidad funcional por daños cerebrales. } \\
\text { C30.-La formación en neuroeducación física permite a los docentes no caer en neuromitos asociados a la educación física. }\end{array}$ \\
\hline
\end{tabular}


calculó el coeficiente de competencia de los mismos siendo de $k=.9$, lo que muestra un nivel de competencia alto (García \& Fernández, 2008; López, 2000; Mengual, 2011). Tras anal izar los cuestionarios de validación se reajustaron algunas preguntas, sin afectar al fondo delacuestión. Por otraparte, seefectuó una prueba piloto a un subgrupo de la muestra para revisar dificultades de comprensión, identificar las preguntas que generaban duda, etc., se utilizó la correspondiente lista de control (Iraossi, 2006). Los resultados de la prueba piloto fueron satisfactorios por lo que se dio por validado el instrumento en su contenido.

Validez de constructo (Análisis Factorial Exploratorio).

La técnica de análisis factorial que hemos aplicado en nuestra investigación sigue las directrices marcadas que establece las siguientes etapas siendo de carácter exploratorio (García-Ferrando, 2000; Díaz de Rada, 2002):

1.-Estudio de la matriz de correlaciones: es necesa rio estudiar la matriz de correlaciones para comprobar si nuestros datos son adecuados para realizar un Análisis Factorial. Para ello, dicha matriz ha de tener una cierta estructura. Para comprobar esto se ha utilizado la medida Kaiser-M eyer-O Ikin de adecuación de muestreo (coeficiente KMO), en nuestro caso el valor es de.771, siguiendo a Kaiser (1974) el valor es aceptable, la prueba de esfericidad de Bartlett tiene una significación de .000 , y el valor de la determinante es $6.064 \mathrm{E}^{-7}$, por lo que continuamos con el análisis.

2. -Extracción de los factores: una vez que se decidió que el análisis factorial puede dar buenos resultados, se procede a la extracción de los factores. En una extracción buena dichos valores deben ser altos (cuanto más próximo a uno mejor) en todas las variables. La tabla de comunalidades resultante nos mostró que los factores tienen un valor superior a. 606 por lo que no es necesa rio eliminar ningún ítem del anál isis factorial. Los ítems mejor representados son: A2 (.797)-LaActividad Física Adaptada es la que se acondiciona para personas con condiciones limitadas como la discapacidad, las deficiencias en la salud o la edad avanzada, para favorecer la integración de estas personas en un estilo de vida saludable a través de la práctica deportiva. A8 (.799)-En todo momento es preferible llevar a cabo la práctica en grupo específico/ exclusivo para las personas con discapacidad. A10 (.798)-Para llevar a cabo sesiones de actividad física adaptada no es necesario realizar adaptaciones curriculares. El ítem peor representado es: B17 (.590)-Las medidas de atención a la diversidad relacio- nadas con los agrupamientos resultan fundamental es para lainclusión educativa del alumnado con diversidad. C25 (.558)-Las clases de Educación Física se convierten en un entorno natural adecuado para observar problemas neuronales asociados al área motriz.

3-Rotación de los factores: existen reglas para saber el número más adecuado de factores a conservar, por ejemplo, la que se conoce como criterio de Kaiser (1974) que indica que hay que conservar los componentes principales cuyos valores propios son mayores que la unidad, aunque el criterio más utilizado es el de observar el porcentaje de varianza total explicada por cada componente 0 factor, y cuando éste llega a un porcentaje acumulado considerado alto, en nuestro caso son los 10 primeros factores, que explican un $69.646 \%$ de la varianza acumulada.

4-Estudio de las puntuaciones factoriales: se muestra en la tabla 2, el análisis de varianza explicada y acumulada, así como determinación de factores y distribución de ítems atendiendo al mayor nivel de saturación por factores.

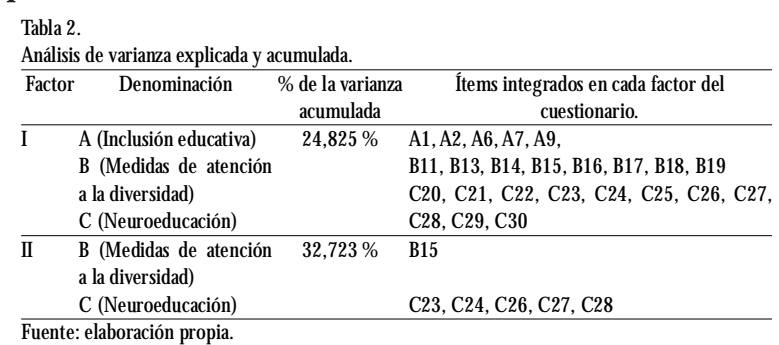

Hemos calculado el alpha de Cronbach de ambos factores: Factor 1: .986 (24 ítems), valoración «excelente». Factor 2: .733 (6 ítems), valoración «aceptable». Tomamos el factor 1, que presenta una fiabilidad más alta que la propia escala original, consiguiendo una escala final de 24 ítems, reduciendo 6 ítems.

Análisis factorial confirmatorio

EI AFC lo realizamos a través de la llamada «metodología SEM » que consta de una serie de fases según Kaplan (2000) y Kline (2005) y que concretamos en cuatro.

Fase I.-Especificación del Modelo de Medición

En esta etapa se establecen los rasgos latentes y las dimensiones que los representan como variables de interés de una teoría sustantiva. Esta etapa es de carácter conceptual y en ella se formula la estructura de relaciones entre las variables latentes representadas por las dimensiones del instrumento y las respuestas a los reactivos del cuestionario de contexto. El Modelo Con- 
ceptual de la escala Likert obtenida del análisis factorial exploratorio está compuesto por 30 ítems observados que se agrupan en tres dimensiones. Para el estudio estructural vamos a utilizar la escala original con 30 variables observadas.

Fase II.-Identificación. Implementación Computacional del Sistema de Ecuaciones Estructura les. Para determinar si el modelo está identificado debemos calcular los grados de libertad, en nuestro caso el valor es de $209 \mathrm{gl}$ por lo que podemos decir que el modelo está sobre-identificado.

\section{Fase Ill.-Estimación de parámetros}

La fase de estimación del modelo incluye una representación gráfica de la estructura teórico-conceptual del instrumento bajo análisis. Esta representación es la base para la formulación de la matriz reproducida que será comparada con la matriz derivada. Para la escala Likert la representación gráfica se muestra en la figura, en la que los óvalos representan las cinco variables latentes (constructos) y los recuadros las 30 variables observa das, que en conjunto conforman la dimensionalidad del instrumento.

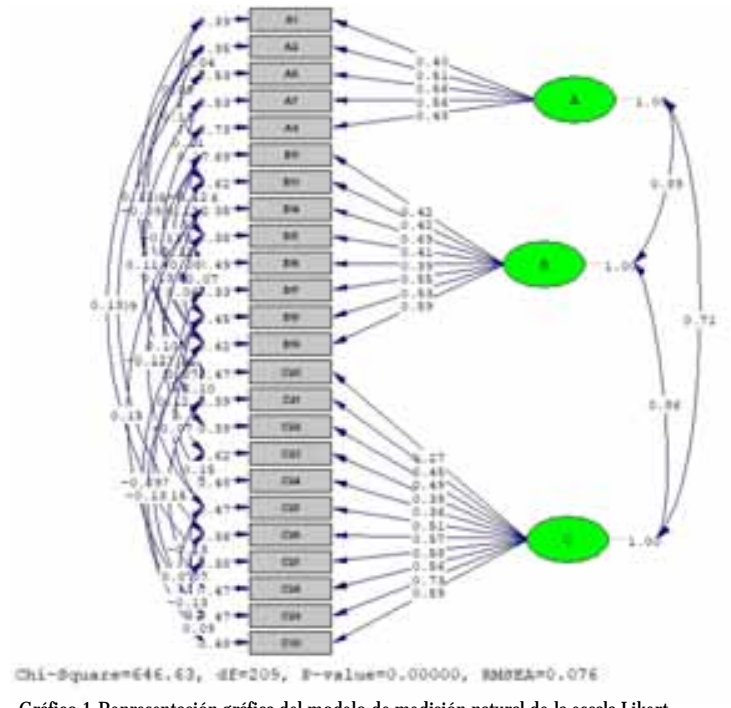

Gráfico 1.Representación gráfica del modelo de medición natural de la escala Likert.

En cuanto a los coeficientes de regresión entre las variables latentes y las observadas, la interpretación es como sigue.

Dimensión A (Inclusión educativa): - Mayor influencia de la variable latente sobre: A6 (.56).-La práctica del Deporte y de laActividad Física en grupo inclusivo es aquella en la cual las personas con discapacidad compartirán todas las actividades físico-deportivas con las personas sin discapacidad. A7 (.56).-La práctica de Deporte y de Actividad Física en grupos específicos/ exclusivos, hace referencia a la práctica de las personas con discapacidad, realizando un trabajo conjunto, al margen del grupo ordinario. -Menor influencia de la variable latente sobre: A1 (.40).-La inclusión educativa es el proceso de análisis sistemático de las culturas, las políticas y las prácticas escolares para tratar eliminar 0 minimizar, a través de iniciativas sostenidas de mejora e innovación escolar.

Dimensión B (M edidas de atención a la diversidad). - M ayor influenciadelavariable latente sobre: B14 (.69).Dentro de las medidas de atención a la diversidad en Educación Física se debe de tener en cuentaal al umnado que presenta discapacidad física transitoria o permanente, discapacidad física grave y permanente. -M enor influenciadela variable latentesobre: B15 (.41). -Las adaptaciones curriculares son un tipo específico de medida de atención a la diversidad.

Dimensión C (Neuroeducación):-M ayor influencia de la variable latente sobre: C29 (.73).-La formación en neuroeducación Física permite a los docentes comprender mejor la situación de las personas con diversidad funcional por daños cerebrales. -M enor influencia de la variable latente sobre: C20 (.27).-La neuroeducación es una línea de pensamiento que tiene por objeto unir los conocimientos del cerebro y el aprendizaje con la psicología cognitiva y la pedagogía.

En resumen, la relación más fuerte entre las varia bles latentes viene dada por:

A (Inclusión educativa) - B (Medidas atención a la diversidad) $->.89$

B (Medidas atención a la diversidad) - C (Neuroeducación) $->.86$

La relación más baja viene dada por:

A (Inclusión educativa) - C (N euroeducación) - >.71

\begin{tabular}{|c|c|}
\hline \multicolumn{2}{|l|}{ Test de $X^{2} y X^{2} / g l$} \\
\hline $\mathrm{X}^{2}$ & 646.63 \\
\hline Grados de libertad & 209 \\
\hline p-valor & 0.000 \\
\hline$x^{2} / g \mid$ & 3.09 \\
\hline \multicolumn{2}{|l|}{ Indices de ajustes absolutos } \\
\hline GFI (Goodness of Fit Index) índice de bondad de ajuste. & .85 \\
\hline $\begin{array}{l}\text { RMSEA (Root Mean Square Error of Aproximation) raíz del residuo cuadrático } \\
\text { promedio de aproximación. }\end{array}$ & .076 \\
\hline N CP (N on Centralized Parameter) índice de parámetro no centralizado. & 437.63(34.80-518.07) \\
\hline $\begin{array}{l}\text { SR MR (Standardized M ean Quadratic Residue) índice estandarizado de residuo } \\
\text { cuadrático medio. }\end{array}$ & .044 \\
\hline RMS (Root Mean Square Residuals) raíz de residuos cuadráticos medios. & .061 \\
\hline ECVI (Expected CrossValidation Index) índice de validación cruzada esperada. & $2.31(2.11-2.54)$ \\
\hline \multicolumn{2}{|l|}{ Indices de ajuste incremental } \\
\hline AGFI (Adjusted Goodness of Fit Index) índice ajustado de calidad de ajuste. & .79 \\
\hline IFI (Incremental Fit Index) índice incremental de ajuste. & .96 \\
\hline NFI (Normed Fit Index) índice de ajuste normalizado. & .94 \\
\hline TLI o N NFI (Tucker-Lewis Index o Non-N ormed Fit Index) índice deTúcker-Lewis & .94 \\
\hline RFI (Relative Fit Index) índice de ajuste relativo. & .92 \\
\hline CFI (Comparative Fit Index) índice de ajuste comparativo. & .96 \\
\hline \multicolumn{2}{|l|}{ Indices de parsimonia } \\
\hline $\begin{array}{l}\text { PNFI (Parsimonius Normed Fit Index) índice de parsimonia de ajuste } \\
\text { normalizado. }\end{array}$ & .71 \\
\hline $\begin{array}{l}\text { PGFI (Parsimonius Goodned of Fit Index) índice de calidad de ajuste } \\
\text { parsimonioso. }\end{array}$ & .60 \\
\hline AGFI (Adjusted Goodness of Fit Index) índice ajustado de bondad de ajuste. & .79 \\
\hline
\end{tabular}


Fase IV.-Evaluación del ajuste. A plicación de índices y criterios de bondad de ajuste

En esta etapa usamos índices y criterios de bondad de ajuste (tabla3) para relacionar la evidencia val idadora con la estructura dimensional del instrumento que se evalúa:

En resumen: $X^{2} / \mathrm{gl}$ (3.09). IAA: GFI (.85), RMSEA (.076), NCP (437.63), SRMR (.44), RMS(.061), ECVI (2.31). IAI:AGFI (.79), IFI (.96), NFI (.94), TLI O N NFI (.94), RFI (.92), CFI (.96). IP: PNFI (.71), PGFI (.60), AGFI (.79)

Como se puede observar se cumplen los criterios de todos los índices de bondad de ajuste, por lo que el modelo está totalmente confirmado.

\section{Resultados}

El resultado más relevante que se deduce de la investigación es la confirmación de la relación entre las tres dimensiones de estudio, aunque con diferente intensidad y matices que discutiremos más adelante.

Como resultado secundario de la investigación, se ha validado en contenido una escala que permite el estudio de la relación entre inclusión educativa, medidas de atención a la diversidad y neuroeducación. EI AFC ha mostrado la validez en el constructo de la misma, mostrando qué ítems son de mayor peso (la educación física es la que se debe adaptar a las personas con discapacidad, mejor utilizar un grupo específico para personas con discapacidad, las adaptaciones curriculares no son tan necesarias como se pensaba), y cuáles son de menor peso (las medidas de atención a la diversidad en agrupamientos no son tan fundamentales para la inclusión, las clases de educación física no son un entorno tan ideal para observar problemas neuronales motores). Igualmente, en las diferentes dimensiones se han determinado qué aspectos son más relevantes y cuáles menos, así en la inclusión educativa los aspectos que destacan son la prácticay actividad en grupo inclusivo, siendo el que menos influencia tiene la cultura o la política. En relación a las medidas de atención a la diversidad, destacan los alumnos con discapacidad física como foco de atención, y con menor importancia las adaptaciones curriculares como medida y solución. Por último, respecto de la neuroeducación, hay que destacar la necesidad de una formación en neuroeducación, sobre física, para comprender mejor la diversidad, no como línea de pensamiento, si no como práctica docente.

Con todo esto, existe según el AFC realizado, una relación fuerte entre las tres dimensiones, inclusión con medidas de atención a la diversidad y ésta con neuroeducación, siendo la relación débil entre inclusión educativa y neuroeducación.

\section{Discusión}

El análisis de los datos, resultante de la investigación, permite afirmar la relación entre las dimensiones de estudios, permitiendo los resultados mostrar los aspectos más y menos influyentes, en el contexto de la educación física, en inclusión educativa, medidas de atención a la diversidad y neuroeducación. Los resultados han permitido tener un instrumento fiable, validado y confirmado para anal izar y seguir investigando estetriángulo de tópicos, pensamos que clave, para la educación física, demostrándose que tenemos una relación entre inclusión y neuroeducación parala atención a la diversidad, orientando en focalizarnos más en que los grupos de alumnos sean inclusivos, en la formación neuroeducativa de los docentes, y no tanto en elaborar adaptaciones curriculares, como respuesta a la diversidad, idea que por otra parte ya se evidencia en Arnaiz (2019) cuando discute sobre el futuro de lainclusión y la necesidad de tomar otros rumbos para su consecución. Los datos obtenidos proceden de estudiantes que en un futuro próximo serán docentes, y aquí radica la limita ción de esta investigación y también el punto de partida, pues quedaría por ampliar el estudio en docentes de educación primaria y contrastar datos para aportar ma yores evidencias.

\section{Conclusión}

La investigación presentada concluye la importancia que tiene la afirmación de que la actividad física adaptada es la que se acondiciona para personas con condiciones limitadas como la discapacidad, las deficiencias en la salud o la edad avanzada, para favorecer la integración de estas personas en un estilo de vida saludable, a través de la práctica deportiva y que para llevar a cabo sesiones de actividad física adaptada no es necesario rea lizar adaptaciones curriculares. Menos importante es que las medidas de atención a la diversidad relacionadas con los agrupamientos resultan fundamentales para la inclusión educativa del alumnado con diversidad, o que las clases de Educación Física se conviertan en un entorno natural adecuado paraobservar problemas neuronales asociados al área motriz. Todos esto, consideramos que es de gran interés para el ámbito de la Educación Física, pues marca una línea de trabajo más práctica y menos 
preocupada por elaborar documentos teóricos que dicen lo que hacer en las aulas, pero no cómo hacerlo. Por otra parte, en el ámbito científico, es de destacar la necesidad de reorientar las investigaciones en temáticas de impacto aportando nuevas visiones y campos de estudio (M újica, 2021; Flores y Maureira, 2020) en la inclusión educativa, las medidas de atención a la diversidad y la neuroeducación.

\section{Referencias}

Abellán, J. (2015).Actitudeshacialadiscapacidad mostradaspor losfuturosmaestrosdeEducación Física. Sportisscientifictechnical Journal, 1,3, 207-219.

Ainscow, M. (2017). Haciendo quelasescuelassean inclusivas leccionesa partir del análisisdelainvestigación internacional. Revista deEducación Inclusiva, 5, 1, 39-49.

Arnaiz, P. (2011). Luchando contralaexclusión: buenasprácticasy éxito escolar. Revista delnnovación Educativa, 21, 23-35.

Arnaiz, P. (2019). La educación indusivaen el sigloXXI.Avancesy desafíos. Murcia: UM.

Arnaiz, P. (2020). ¿Esposibleunaenseñanza100\% equitativa? Laverdad. E, octubre, 2020

Battro,A.M ., \& Cardinali, D.P (1996). Máscerebro en la educación. Buenos Aires: LaNación.

Booth, T., \&Ainscow, M. (Eds.). (1998). FromThemTo U s.An International Study of Indusion in Education. London: Routledge.

Campos, A. (2010). Neurociencia: uniendo lasneurocienciasylaeduca ción en labúsquedadel desar rollo humano. La Educación, 1, 1-14.

DíazdeRada,V. (2002). Tiposdeencuestasy diseñosdeinvestigación. Pamplona: Universidad PúblicadeN avarra.

Echeita, G., \&Ainscow, M. (2011). La educación inclusiva como derecho. Marco dereferencia y pautasdeacción para el desarrollo deuna revolución pendiente. Tejuelo: Didácticadelalenguaylaliterđura. Educación, 1, 12, $26-46$.

Flores, E., \& M aureira, F. (2020). Formación pedagógicaen lacarrerade Educación Física: faltade conocimientosparaun profesional del siglo XXI. EmásF, Revista Digital deEducación Físca, 11,62-70.

Flores, G. (2019). jugamos al Súper Mario Bros? Descripción de una experienciagamificadaen laformación del profesorado deEducación Física Retos N uevastendenciasen Educación Física, Deportey Recreación, 36, 529-534.

García, L., \& Fernández, S. (2008). Procedimiento deaplicación del traba jo creativo en grupo deexpertos. Energética, 29, 2, 46-50.

GarcíaFerrando, M. (2015). El análisisdela realidad sodal: métodosytécnicasde investigación. Madrid:Alianzaeditorial.

GeorgeD., \& MalleryP. (2003). SPSS folW indowstep by step:A simpleguideand reference. 11.0 update. Boston: Allyn \& Bacon.

González-López, I., \& Macía-García, D. (2018). Laformación permanentecomo herramientaparamejorar laintervención del mæestro de educaciónfísicaconalumnado con discapacidad. Retos, 33, 118-122. https:/ / doi.org/ 10.47197/ retos. v0i33.55056

Guba, E.G. (1990). Theparadigm dialog. London:Sage.

Hernández, F.J., Labrador,V., Niort.J., Berbel, G. \&Trullols, M. (2017). Respuestas del profesorado de Educación Física ante conflictos con alumnado con discapacidad intelectual yfísica. Retos. Nuevastendencias en Educación Fíśca, Deportey Recreación, 31, 123-127.

Iraossi, G. (2006). ThePower of Survey Design:A U ser's Guidefor M anaging Surveys Interpreing Results and Influencing Respondents Washington, D. C.:The World Bank.

Kaiser, H. F. (1974).An index of factorial simplicity. Psychomerika, 39, 31-
36

Kaplan, D. (2000). Structural equation modeling: Foundations and extensions. Thousand 0 aks, CA: SagePublications.

Kinio, A., Dufresne, L., Brandys, T., \& Jetty, P. (2017). Break out of the classroom: Theuse of escaperoomsasan alternativelearningstrategy for surgical education. Journal ofVascular Surgery, 66, (3), e76.

Kline, R. (2005). Principlesand pradicesof strucural equation modding (2ned.). NewYork: Guilford Press.

Koizumi, H. (2008). Developing the brain: A functional-imaging based approachto learningand eductional sciences. In Battro,A. M., Fischer K.W., \& Léna, P.J (Editors). TheEducated Brain. Cambridge: Cambridge University Press.

López,A. (2008). Lamoderación de lahabilidad diagnóstico patológico desdeel enfoquehistórico cultural paralaasignaturaPatologíaVeterinaria. Revista Pedagógica U niversitaria, 13, 5, 51-71.

M aureira, F. (2018). PrincipiosdeN auroeducación Físca (2 ededición). La Nueva Ciencia Del Ejercicio. Madrid, España: Bubok PublishingSL.

Melguizo-Ibánez, E., ZuritaO rtega, F., U bago-Jiménez, J., \& GonzálezValero, G. (2020). Niveles de adherencia a la dieta mediterránea e inteligenciaemocional en estudiantes del tercer ciclo de educación primaria de la provincia de Granada. Retos, 40, 264-271. https: / doi.org/ 10.47197/ retos.v1i40.82997

Mengual, S. (2011). La importancia percibida por e profesorado y el alumnado sobrela inclusión dela competencia digital en educación Superior (Tesisdoctoral, Universidad deAlicante).

MujicaJ ohnson, F. (2021). Emocionesnegativasdel alumnado de Secundariaen el aprendizaje de baloncesto en Educación Física. Retos, 41, 362-372. https: / doi.org/ 10.47197/ retos. v0i41.84395

MujicaJ ohnson, F., \& O rellanaA Arduiz, N. (2020). Emocionesdel profesorado deeducaciónfísica: revisiónnarrativa(2010-2020). Retos, 39, 910-914. https: / doi.org/ 10.47197/ retos. v0i39.80750

M untaner, J.J. (2019). Lainclusión una apuesta social y educativa, en El Homrani, M., Arias, S.M \& \& Ávalos, I. (Coords.). La inclusión: una apuesta educativay social. (41-57). Madrid:WoltersK luwer.

O choa-M artínez, P., Hall-López, J., CarmonaLópez,A., Reyes Castro, Z., Sáenz-López Buñuel, P., \& Conde-García, C. (2019). Análisis comparativo deun programaeducaciónfísicaen niños con discapacidad auditivasobrelaedad motoraequivalente. Retos, 35, 310-313. https:/ / doi.org/ 10.47197/ retos. voi35.67190

Palomares, A. (Coord) (2020). Investigación y respuestas socioeducativas del profesorado en el sigloXXI. Madrid: Síntesis.

Pastas, H.I. (2018). Lostegidosdela Educación Físca intercultural: fortaleciendoel pensamientoancestral indígena (Trabajo Fin de Grado). Universidad Peda gógicaN acional de Colombia, Bogotá, D. C.

Pérez-Enseñđa, A., \& M oyaz Mata, I. (2020). Diversidad en laidentidady expresión degénero enEducaciónFísica: Unarevisión delaliteratura. Retos, 38, 38, 818-823. https:// doi.org/ 10.47197/ retos.v38i38.54076

Ricoy, C. (2006). Contribución sobre los paradigmas de investigación. EducaçãoSanta María, 31, (01), 11-22.

Segura,A., \& Parra, M.E. (2019). How to implement activemethodologies inPhysical Eduction: Escape Room. Education, Sport, Health and Physical Adivity, 3, (2), 295-306.

Silva, S. (2007). Atención a la diveriddad:necesidadeseducativas,guía deaduación para docentes Vigo: Ideaspropiaseditorial.

Tenorio, J. (2013). Representaciones sociales sobre el profeserado de Educación Físca. TesisdeM aestría, U niversidadVeracruzana. Xalapa, M éxico.

Valencia-Peris, A., Mínguez-Alfaro, P., \& Martos-García, D. (2019). La formación inicial del profesorado de Educación Física: una mirada desde la atención a la diversidad. Retos, 37, 37, 597-604. https:/ / doi.org/ 10.47197/ retos.v37i37.74180 